\title{
Fever of unknown origin in a Mediterranean survey from a division of internal medicine: report of 91 cases during a 12-year-period (1991-2002)
}

\author{
Pasquale Mansueto - Gabriele Di Lorenzo - Manfredi Rizzo $\cdot$ Salvatore Di Rosa • \\ Giustina Vitale - GiovamBattista Rini - Serafino Mansueto - Mario Affronti
}

Received: 29 June 2007 / Accepted: 3 December 2007

(C) SIMI 2008

\begin{abstract}
Despite the availability of all advanced diagnostic tools, fever of unknown origin (FUO) remains a diagnostic challenge for physicians. The objective was to define, through a retrospective study, the categories of the diseases of Sicilian patients admitted at the Department of Clinical Medicine and Emerging Diseases, University of Palermo, Italy, for classical FUO. Using the registration system for patients admitted from 1991 to 2002, 508 charts of patients admitted because of fever were reviewed. Of these, only 91 patients fulfilled the criteria for classical FUO. The origin of FUO was diagnosed in 62 (68.1\%) patients. Infection was the most common cause of FUO with 29 cases (31.8\% of total of FUO), neoplasms accounted for 13 cases $(14.2 \%)$, collagen vascular disease for 11 cases (12.0\%), and miscellaneous for 9 cases $(9.8 \%)$. Undiagnosed FUO were 29 (31.8\%) and, of them, 22 cases were followed-up for 2 years. A definite diagnosis could be established only in 8 cases, 13 subjects completely recovered and 4 of them died. In the $73.4 \%$ of cases, the FUO have been the result of misleading factors in the diagnostic approaches as made by the physician. The results of our study are similar to those already reported by other authors in other populations, with infections as first, neoplasm as second, and collagen vascular diseases as third most important causes of FUO. In our study the prognosis for
\end{abstract}

P. Mansueto $(\square) \cdot$ G. Di Lorenzo · M. Rizzo · G. Vitale ·

G. Rini - S. Mansueto - M. Affronti

Dipartimento di Medicina Clinica e delle Patologie Emergenti,

Università di Palermo, Via del Vespro, 141,

90127 Palermo, Italy

e-mail: pamansu@unipa.it

S. Di Rosa

Divisione di Medicina Interna, Villa Sofia Whitaker, CTO,

Palermo, Italy undiagnosed FUO cases was good, but a definite diagnosis could be established only in few cases. Therefore, further multicentric, prospective studies of good design are required.

Keywords Fever of unknown origin - Infection . Neoplasm · Collagen vascular disease $\cdot$ Follow-up

\section{Introduction}

Despite all advanced diagnostic techniques available nowadays, fever of unknown origin (FUO), remains a diagnostic challenge for physicians, especially those working in Internal Medicine Divisions, where this kind of patients are often hospitalized.

FUO has been defined by Petersdorf and Beeson [1], in 1961 , as an illness with temperature exceeding $38.3^{\circ} \mathrm{C}$ on at least three occasions, lasting at least 3 weeks, with no diagnosis reached after 1 week of intensive investigation. In recent years, Durack and Street [2] have suggested classifying patients with FUO into four categories: classical, neutropenic and, nosocomial FUO, and FUO associated with HIV. Each group has a unique differential diagnosis based on characteristics and vulnerabilities, and, therefore, a different process of evaluation. The revised criteria require an evaluation of at least three days in the hospital, or three outpatient visits, or one week of logical and intensive outpatient testing, without clarification of the fever's cause.

When dealing with such disease, it is important that causes of FUO and their relative frequencies in the population should be known. Thus, for the diagnosis of FUO, it is appropriate to consider the most frequent diseases first, rather than the rare ones. The diagnostic process should be 
guided by potential diagnostic clues (PDCs), retrieved by meticulous approaches, including history taking, physical examination, and baseline tests. Directed diagnostic workup, using the PDCs, remains the most efficient way to solve the problem of FUO. A standardized flow chart can be applied in the absence of PDCs or when the PDCs are contradictory [3-8].

FUO's diagnostic approach always includes a comprehensive history, a careful, repeated, physical examination, appropriate laboratory tests and radiographic/endoscopic studies. More invasive testing, such as bone marrow aspiration or biopsy, lymph nodes and liver biopsy, should be performed only when clinical suspicion shows that these tests are indicated or when the source of the fever remain unidentified after extensive evaluation $[8,9]$.

The development of microbiological tests and radiodiagnostic procedures influenced the distribution of the causes of FUO. Additionally, factors, such as geographic factors, the age of the patients, the experience of researchers, and the period of the study, might affect the distribution of the diagnostic categories. Therefore, it is difficult to compare patients affected by FUO in different populations $[5,6]$.

The most common causes of classic FUO are infections, malignancies and collagen vascular diseases, but there are well over 200 different reported causes of FUO [3, 4]. In most FUO series, infections were in the majority with a relative frequency ranging from 21 to $58 \%$, with, in recent years, an increase in the proportion of collagen vascular diseases [1, 5, 9-23]. The spectrum of diseases seems to be determined by geographic and economics factors, as well as by hospital setting and clinical practice of the physicians $[1,5,9-23]$.

The purpose of the present study was to define, through a retrospective study, the categories of the diseases of Sicilian patients admitted in a Medical Department for classical FUO, as defined by Petersdorf and Beeson [1], revised by Durack and Street [2].

\section{Methods}

The present study has been approved by the ethics committee of our Dipartimento di Medicina Clinica e delle Patologie Emergenti, University of Palermo, Italy. Our department is a University-based referral centre, which is located in a region (Sicily) where about 5 million people live. Using the registration system for adult patients admitted to our department, examined at the Emergency Department, or at the Internal Medicine Outpatient Clinic, of the same Dipartimento, from 1991 to 2002, 508 charts of patients admitted because of fever were reviewed, among a total of 4,585 (11.0\% of all admissions). Of these, only 91 patients fulfilled the criteria of Petersdorf and Beeson [1], revised by Durack and Street [2], for classical FUO, (1.9\% of all admissions and $17.9 \%$ of all admissions because of fever). In this study, the patients admitted because of fever were often referred to our department from different hospitals of the region, where their FUO was not diagnosed.

Patients with a known history of neutropenia, nosocomial infections and immunosuppressive diseases (e.g., HIV positive patient) were excluded. In all patients, with a diagnosis of classical FUO, clinical history, physical examination, and the following tests were performed, as a first step: erythrocyte sedimentation rate, $\mathrm{C}$ reactive protein, hemoglobin, mean cellular volume, platelet count, absolute and differential leukocyte count, $\mathrm{LDH}$, serum urea nitrogen, creatinine, serum sodium, serum potassium, protein fractions, and alkaline phosphatase, urinary analysis, fecal occult blood (at least three times), HIV testing, antistreptolysin $\mathrm{O}$ titer, tuberculin skin testing, blood (at least three times), urine, feces and sputum cultures, chest $\mathrm{X}$-ray and ultrasonography of the abdomen. According to their history, physical examination, and laboratory findings, in the following days, the following tests were performed, as a second step: serology for Cytomegalovirus, Epstein-Barr virus, Mycoplasma, Chlamydia, Brucella, Salmonella, Toxoplasma, Leishmania, hepatitis viruses, rheumatoid factor, antinuclear antibodies, serum levels of CH50, C3 and C4, TSH, fT4, fT3, thyroglobulin antibodies and thyroperoxidase antibodies, immunoelectrophoresis of serum, sputum for acid-fast bacilli, polymerase chain reaction and urine cultures for tuberculosis, radiography of sinus, orthopantomography, echocardiography, and computed tomography of chest and abdomen. Invasive procedures, such as esophagogastroduodenoscopy, colonoscopy, bone marrow aspiration or biopsy, lymph nodes and liver biopsy, were performed only when a diagnosis was not revealed by the non-invasive procedures (Table 1).

Final diagnoses reported in this study were made by the authors upon critical revision of the data and diagnosis mentioned in medical records. The causes of FUO were classified into five groups: infections, neoplasms, collagen vascular diseases, other causes (miscellaneous), and undiagnosed. Undiagnosed cases with FUO were followed-up for 2 years.

We also compared the prevalence of each cause of FUO found in the present study and previously published data, using MEDLINE ${ }^{\circledR}$ database search and manual research on Internal Medicine specialized journals.

Chi-squared test (for nominal variables) was performed (using Stat View ${ }^{\circledR} 5.0$ software, SAS Institute, Cary, NC, USA) to assess possible significant differences in the prevalence of each cause of FUO between the present study and previous published data. 
Table 1 First line, second line and third line exams for FUO

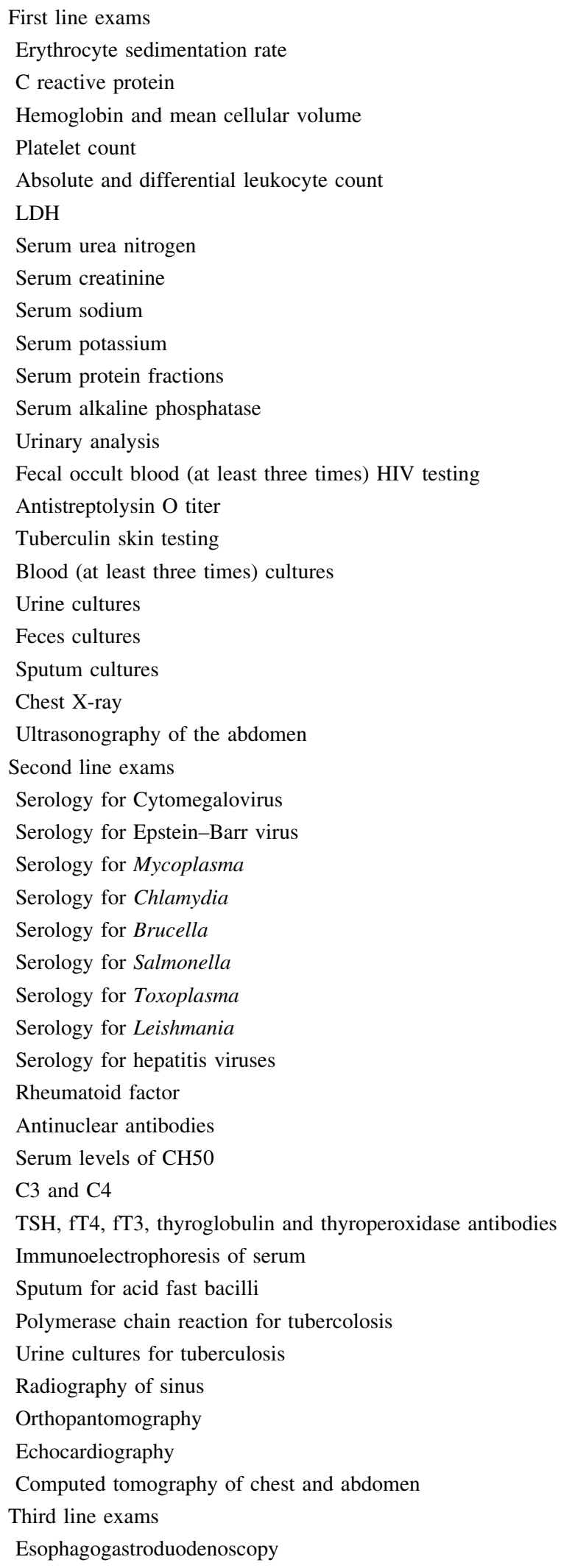

Table 1 continued

\section{Colonoscopy}

Bone marrow aspiration or biopsy

Lymph nodes biopsy

Liver biopsy

Finally, we retrospectively analyzed the concomitant misleading factors that generated a diagnosis of FUO, classifying them in five different categories: (1) Rarity and peculiarity of the clinical pattern; (2) mistakes regarding the anamnesis; (3) mistakes regarding the clinical examination; (4) wrong interpretation of a laboratory test, and (5) inadequete evaluation of a symptom and/or a positive test.

\section{Results}

We evaluated 91 patients (54 males and 37 females), mean age of 47.9 years (range 14-74), with a median duration of hospitalization of 22.3 days (range 8-102). In our series, a cause of FUO was established in $62(68.1 \%)$ patients. Infection was the most common cause of FUO, in 29 cases ( $31.8 \%$ of total FUO); neoplasms accounted for 13 cases (14.2\%); collagen vascular disease were the cause of fever in 11 patients $(12.0 \%)$, and miscellaneous in nine cases (9.8\%). Undiagnosed FUO were 29 (31.8). Among infections, the most important causes of FUO were represented by abdominal abscesses (seven cases), pulmonary (one case) and extra-pulmonary tuberculosis (three cases), Cytomegalovirus infection (three cases), hepatocholangitis (three cases), and visceral leishmaniasis (three cases); among neoplasms by lymphoma (six cases); among collagen vascular diseases by rheumatoid arthritis (three cases); and, finally, among miscellaneous by Crohn disease (two cases) (Table 2).

We also made a comparison between the prevalence of each cause of FUO found in the present study and previous published data (Table 3). In general, we found that our data were consistent to those already reported, but more specifically, for infections we found significant differences $(P<0.05)$ with Traverso et al. [16], Shoji et al. [20] and Campanella et al. [21]; for neoplasms with Larson et al. [11] and Howard et al. [14]; for collagen vascular diseases with Iikuni et al. [18], and Sica et al. [22], and for undiagnosed FUO with Petersdorf and Beeson [1], Jacoby and Swartz [10], Kanzanjian [12], Howard et al. [14], and Sica et al. [22].

Twenty-nine subjects (31.8\%) were discharged without a final diagnosis being established. Seven subjects were not traceable. The follow-up duration of the other 22 subjects 
Table 2 Infective and non-infective causes of fever of unknown origin and diagnosis

\begin{tabular}{|c|c|c|}
\hline Diagnostic categories & $\begin{array}{l}\text { No of patients } \\
(n=91)\end{array}$ & $\%$ \\
\hline Infections & 29 & 31.8 \\
\hline Abscesses & 7 & 7.6 \\
\hline Tuberculosis & 4 & 4.3 \\
\hline CMV infection & 3 & 3.2 \\
\hline Hepatocholangitis & 3 & 3.2 \\
\hline Visceral leishmaniasis & 3 & 3.2 \\
\hline Chlamydia infection & 2 & 2.1 \\
\hline $\begin{array}{l}\text { Hydatidosis, salmonellosis, } \\
\text { pharyngitis, leptospirosis, } \\
\text { staphylococcosis, rickettsiosis, } \\
\text { Mycoplasma pneumoniae infection }\end{array}$ & 1 & 1.0 \\
\hline Neoplasms & 13 & 14.2 \\
\hline Lymphoma & 6 & 6.5 \\
\hline Hepatobiliar cancer & 2 & 2.1 \\
\hline Colon cancer & 2 & 2.1 \\
\hline Acute leukaemia & 1 & 1.0 \\
\hline Pulmonary cancer & 1 & 1.0 \\
\hline Renal cancer & 1 & 1.0 \\
\hline Collagen vascular diseases & 11 & 12.0 \\
\hline Rheumatoid arthritis & 3 & 3.2 \\
\hline Systemic lupus erythematosus & 2 & 2.1 \\
\hline Polymyalgia rheumatica & 2 & 2.1 \\
\hline Systemic vasculitis & 2 & 2.1 \\
\hline Adult Still's disease & 1 & 1.0 \\
\hline Henoch-Schonlein purpura & 1 & 1.0 \\
\hline Miscellaneous & 9 & 9.8 \\
\hline Crohn disease & 2 & 2.1 \\
\hline Drug fever & 1 & 1.0 \\
\hline Familial mediterranean fever & 1 & 1.0 \\
\hline Pressure ulcers & 1 & 1.0 \\
\hline Ovarian cyst & 1 & 1.0 \\
\hline Granulomatous hepatitis & 1 & 1.0 \\
\hline Sarcoidosis & 1 & 1.0 \\
\hline Thrombophlebitis & 1 & 1.0 \\
\hline No diagnosis & 29 & 31.8 \\
\hline
\end{tabular}

was 48 months (Table 4). A definite diagnosis could be established only in 8/22 cases, 3/8 within 2 months after discharge. The diagnoses were: Cytomegalovirus infection, infective endocarditis, brucellosis, chronic calculous cholecystitis, underwent cholecystectomy, and chronic tonsillitis, underwent tonsillectomy. Thirteen individuals became symptom free following discharge, 7/13 within 2 months after discharge, and were considered to be finally cured. Four patients died, and the cause of death was considered to be related to the disease that caused FUO in three cases (metastatic colon cancer, metastatic lesions from an unknown primary tumor, lymphoma). One patient died from upper gastrointestinal bleeding associated with the use of nonsteroidal anti-inflammatory drugs (NSAIDs).

In our study, the FUO have been the result of several concomitant misleading factors: the rarity and peculiarity of the clinical pattern (13.3\% of cases), mistakes regarding the anamnesis $(24.5 \%)$, and the clinical examination $(22.6 \%)$, the wrong interpretation of a laboratory test $(20.7 \%)$, and inadequecy in the evaluation of a symptom and/or a positive test $(5.6 \%)$. Taken together, a clinical mistake was present in the $73.4 \%$ of cases.

\section{Discussion}

Causes of FUO are generally determined by infections (20-58\%), neoplasms (6-31\%), collagen vascular diseases $(8-31 \%)$, or other causes (4-23\%). There may be also no definitive diagnosis (4-38\%) in some cases (Table 2) [1, 5, 9-23]. In agreement with such previous reports, the most frequent causes of FUO in our study were infections $(31.8 \%)$, followed by neoplasms (14.2\%) and collagen vascular diseases (12.0\%). These findings suggest that FUO may have similar prevalence in populations with different ethnic and geographical backgrounds.

In our study, causes of FUO were usually either unusual presentation of a well-known disease, namely, a common disease with a unique symptom, fever, or more rarely, an uncommon condition. Other symptoms and/or signs either did not appear or appeared later.

Among infections associated with FUO, abscesses, tuberculosis and Cytomegalovirus infection, were the most common; this was the case in other Italian studies too [16, $17,21,22]$. Other causes of infections, such as hepatocholangitis, visceral leishmaniasis and Chlamydia infection were identified in our patients with FUO, but with unusual presentation. In our study, intraabdominal abscesses, often associated with diverticulitis, were found in seven patients. Three of these patients were older than 70 years. Elderly patients typically have a more subacute course, with few symptoms and signs, and a long illness, as was the case in our patients [24]. Extra-pulmonary tuberculosis caused special diagnostic problems. The difficulties were disseminated diseases, without the characteristic miliary pattern on chest X-ray, or without clear localized lesions, with negative sputum smears and tuberculin skin test. Rapid diagnostic tests in body fluid, such as polymerase chain reaction, were helpful in making the diagnosis. Bacteriological cultures and histopathological investigations were also important in confirming the diagnosis [25, 26]. The diagnosis of leishmania infection, in three cases of FUO, was established in subjects co-affected with hepatic cirrhosis, without significant neutropenia (neutrophil count $>500 / \mu \mathrm{L}$ ), in which the 
Table 3 Etiologic fluctuation of fever of unknown origin. Data are shown as percentual values

\begin{tabular}{|c|c|c|c|c|c|c|}
\hline Publication date & Author & Infections & Neoplasms & $\begin{array}{l}\text { Collagen } \\
\text { vascular } \\
\text { diseases }\end{array}$ & Miscellaneous & Undiagnosed \\
\hline 1961 & Petersdorf and Beeson [1] (100 cases, 1952-1957) & 36 & 19 & 15 & 23 & $7 *$ \\
\hline 1963 & Sheon and van Ommen [13] (60 cases, 1959-1960) & 22 & 17 & 13 & 10 & 38 \\
\hline 1973 & Jacoby and Swartz [10] (128 cases, 1957-1970) & 40 & 20 & 15 & 17 & $8^{*}$ \\
\hline 1977 & Howard et al. [14] (100 cases, 1969-1976) & 37 & $31^{*}$ & 19 & 8 & $5^{*}$ \\
\hline 1982 & Larson et al. [11] (105 cases, 1970-1980) & 30 & $31^{*}$ & 9 & 17 & 13 \\
\hline 1992 & Barbado et al. [15] (133 cases, 1968-1989) & 31 & 18 & 13 & 17 & 21 \\
\hline 1992 & Knockaert et al. [9]. (199 cases, 1980-1989) & 23 & 7 & 21 & 23 & 26 \\
\hline 1992 & Kanzanjian [12] (86 cases, 1984-1990) & 33 & 24 & 16 & 18 & $9 *$ \\
\hline 1992 & Traverso et al. [16] (48 cases, 1980-1990) & $52 *$ & 10 & 8 & 19 & 11 \\
\hline 1992 & Pauluzzi et al. [17] (48 cases, 1971-1990) & 44 & 10 & 21 & 13 & 12 \\
\hline 1994 & Iikuni et al. [18] (153 cases, 1982-1992) & 29 & 14 & $29^{*}$ & 16 & 12 \\
\hline 1995 & $\begin{array}{l}\text { De Kleijn and van der Meer [19] (53 cases, } \\
\text { 1988-1992) }\end{array}$ & 21 & 19 & 23 & 7 & 30 \\
\hline 1995 & Shoji et al. [20]. (80 cases, 1986-1992) & $54 *$ & 9 & 16 & 4 & 17 \\
\hline 1997 & De Kleijn et al. [5] (167 cases, 1992-1994) & 26 & 13 & 24 & 7 & 30 \\
\hline 1998 & Campanella et al. [21] (53 cases, 1990-1994) & $58 *$ & 6 & 15 & 4 & 17 \\
\hline 1999 & Sica et al. [22] (169 cases, 1990-1998) & 36 & 15 & $31^{*}$ & 14 & $4^{*}$ \\
\hline \multirow[t]{2}{*}{2003} & $\begin{array}{l}\text { Vanderschueren et al. [23] (192 cases, } \\
\text { 1990-1999) }\end{array}$ & 20 & 10 & 23 & 13 & 34 \\
\hline & Present study (91 cases, 1991-2002) & 32 & 14 & 12 & 10 & 32 \\
\hline
\end{tabular}

Data are shown as percentual values

* $P<0.05$ vs present study

findings of cachexia, hepatosplenomegaly, peripheral pancytopenia, and hypergammaglobulinemia were first attributed to the hepatic disease. In these cases, diagnosis,

Table 4 Outcome of 22 patients with FUO discharged without a final diagnosis

\begin{tabular}{lcr}
\hline Outcome & $\begin{array}{l}\text { No of } \\
\text { patients }\end{array}$ & \\
\hline Diagnosed & 5 & 22.7 \\
Cytomegalovirus infection & 1 & 4.5 \\
Infective endocarditis & 1 & 4.5 \\
Brucellosis & 1 & 4.5 \\
Chronic calculous cholecystitis & 1 & 4.5 \\
Chronic tonsillitis & 1 & 4.5 \\
Undiagnosed but cured & 13 & 59.0 \\
Dead & 4 & 18.1 \\
Related to FUO & 3 & 13.6 \\
Metastatic colon cancer & 1 & 4.5 \\
Metastatic lesions from an unknown primary tumor & 1 & 4.5 \\
Lymphoma & 1 & 4.5 \\
Not related to FUO & 1 & 4.5 \\
Upper gastrointestinal bleeding by use of NSAIDs & 1 & 4.5 \\
\hline
\end{tabular}

suspected for prolonged elevated fever, cachexia, and high prevalence of leishmania infection in our region, required serology for Leishmania and bone marrow biopsy. In our study, as in other developed countries, viral infections, especially herpes-virus infections, were an important cause of FUO. Cytomegalovirus infection was defined in three cases, all without the typical clinical presentation of the disease (sore throat, pharyngeal erythema, adenopathy and splenomegaly), but only with reactive lymphocytosis and moderately increased serum transaminase. Diagnosis was confirmed by repeated serological testing (CMV-IgM).

Because of substantial increase in the elderly population, as well as advances in the diagnosis and treatment of diseases common in this population, malignancy has become a common etiologic consideration in elderly patients. In our study we found that neoplasms were the second most important cause of FUO. As expected, some hematological malignancies (lymphoma) and tumours of the digestive tract (colon and hepatobiliary cancer) remain difficult to diagnose and therefore remain a cause of FUO, because of lack of localizing symptoms or difficulty in obtaining appropriate biopsies. Nevertheless, the use of CT and MRI imaging have allowed tumors to be found more easily in the last decade [5-7]. 
Collagen vascular diseases, which include connective tissue diseases and vasculitis, were the third most important cause of FUO in our study. Fever preceded more typical manifestations and serological evidence by weeks or months. In particular, rheumatoid arthritis, systemic lupus erythematosus, polymyalgia rheumatica, without associated temporal arteritis, and systemic vasculitis, were the first line causes of this subgroup of FUO, while we found a low incidence of adult Still's disease (one case), in contrast to the data shown by other authors [20, 27]. Among miscellaneous, the two cases of Crohn disease reported intermittent or no bowel symptoms. Careful examination of the abdomen and radiological and endoscopic investigations revealed the diagnosis.

Regarding undiagnosed FUO, its prevalence has always been around $20 \%$ during the years since the study of Petersdorf and Beeson (1961) [1] to the most recent one of Vanderschueren et al. (2003) [23], despite the progress in laboratory and radiological technologies, as well as the attention and experience of researchers.

In our study, the number of undiagnosed cases at discharge was higher than in others $[1,10,12,14,22]$. Twenty-two undiagnosed cases with FUO were followedup for 2 years. A definite diagnosis could be established only in eight cases, 13 subjects completely recovered, and only four of them died. Therefore, outcome of undiagnosed FUO is generally good (81.7\%), hence waiting for PDCs to appear is probably better than ordering more screening investigations, in the hope that something abnormal will comeup $[1,5,11,18,28]$.

In our study, the FUO have been the result of several concomitant misleading factors in the diagnostic approaches made by the physician; regarding the anamnesis $(24.6 \%)$, the clinical examination $(22.6 \%)$, the wrong interpretation of a laboratory test (20.7\%), and inadequecy in the evaluation of a symptom and/or a positive test (5.6\%). This means that three times out of four the FUO was the result of misleading factors in the diagnostic approaches as made by the physician, who might skim over the anamnesis, not be careful in the clinical examination, and not correctly apply the diagnostic methodology, including the laboratory tests. We also cannot exclude the existence of individual genetic factors, influencing the atypical and silent course of undiagnosable illnesses.

The principal limits of our study is that it was a singlecentre, retrospective one. Further multi-centre, prospective studies, of good design, are required. Because of the diversity of patients with FUO, prospective studies are needed that either are large enough and/or focus on certain subgroups.

In conclusion, the results of our study are similar to those already reported by other authors in other populations, with different ethnic and geographical backgrounds, with infections as first, neoplasm as second, and collagen vascular diseases as third most important causes of FUO. In our study the prognosis for undiagnosed FUO cases was good, but a definite diagnosis could be established only in few cases. Therefore, further multicentric, prospective studies of good design are required.

\section{References}

1. Petersdorf RG, Beeson PB (1961) Fever of unexplained origin: report on 100 cases. Medicine 40:1-30

2. Durack DT, Street AC (1991) Fever of unknown origin-reexamined and redefined. Curr Clin Top Infect Dis 11:35-51

3. Arnow PM, Flaherty JP (1997) Fever of unknown origin. Lancet 350:575-580

4. Hirschmann JV (1997) Fever of unknown origin. Clin Infect Dis 24:291-300

5. De Kleijn EM, Vandenbroucke JP, van der Meer JW (1997) Fever of unknown origin (FUO). I. A prospective multicenter study of 167 patients with FUO, using fixed epidemiologic entry criteria. The Netherlands Study Group. Medicine 76:392-400

6. Gaeta GB, Fusco FM, Nardiello S (2006) Fever of unknown origin: a systematic review of the literature for 1995-2004. Nucl Med Commun 27:205-211

7. Petersdorf RG (1992) Fever of unknown origin. An old friend revisited. Arch Intern Med 152:21-22

8. De Kleijn EM, van Lier HJ, van der Meer JW (1997) Fever of unknown origin (FUO). II. Diagnostic procedures in a prospective multicenter study of 167 patients. The Netherlands FUO Study Group. Medicine 76:401-414

9. Knockaert DC, Vanneste LJ, Vanneste SB, Bobbaers HJ (1992) Fever of unknown origin in the 1980s. An update of the diagnostic spectrum. Arch Intern Med 152:51-55

10. Jacoby GA, Swartz MN (1973) Fever of undetermined origin. N Engl J Med 289:1407-1410

11. Larson EB, Featherstone HJ, Petersdorf RG (1982) Fever of undetermined origin: diagnosis and follow-up of 105 cases, 1970-1980. Medicine 61:269-292

12. Kazanjian PH (1992) Fever of unknown origin: review of 86 patients treated in community hospitals. Clin Infect Dis 15:968973

13. Sheon RP, van Ommen RA (1963) Fever of obscure origin. Diagnosis and treatment based on a series of sixty cases. Am J Med 34:486-499

14. Howard P Jr, Hahn HH, Palmer RL, Hardin WJ (1976) Diagnostic evaluation of patients with fever of unknown origin. South Med J 69:933-934

15. Barbado FJ, Vazquez JJ, Pena JM, Arnalich F, Ortiz-Vazquez J (1992) Pyrexia of unknown origin: changing spectrum of diseases in two consecutive series. Postgrad Med J 68:884-887

16. Traverso A, Tassara A, Chasseur R, Longo G, Rossi P, Ansermin A (1992) Febbre di origine indeterminata: diagnosi e follow-up di 48 casi del decennio 1980-1990. Giornale di Malattie Infettive e Parassitarie 44:271-277

17. Pauluzzi S, Fiorio F, Pasticci MB, Cassano F, De Socio G, Tascini C (1992) Le febbri infettive di difficili diagnosi nell'ospite immunocompetente. Giornale di Malattie Infettive e Parassitarie 44:894-900

18. Iikuni Y, Okada J, Kondo H, Kashiwazaki S (1994) Current fever of unknown origin 1982-1992. Intern Med 33:67-73

19. De Kleijn EM, van der Meer JW (1995) Fever of unknown origin (FUO): report on 53 patients in a Dutch university hospital. Neth J Med 47:54-60 
20. Shoji S, Imamura A, Imai Y et al (1994) Fever of unknown origin: a review of 80 patients from the Shin'etsu area of Japan from 1986 to 1992. Intern Med 33:74-76

21. Campanella N, Pergolini M, Daher W, Moraca A, Borgognoni C, Morosini P (1998) Fever of unknown origin. Comparison of the diagnostic spectrum of 53 cases in a medical ward in an Italian hospital with those of other 9 countries. Recenti Prog Med 89:372-376

22. Sica S, Avino S, Glielmi G, Cicero SR (1999) Incidenza di febbri di difficile diagnosi (FDD) osservate dall'1-90 all'8-98. Le Infezioni in Medicina 2:96-104

23. Vanderschueren S, Knockaert D, Adriaenssens T et al (2003) From prolonged febrile illness to fever of unknown origin: the challenge continues. Arch Intern Med 163:1033-1041
24. Norman DC, Yoshikawa TT (1984) Intraabdominal infection: diagnosis and treatment in the elderly patient. Gerontology 30:327-338

25. Elder NC (1992) Extrapulmonary tuberculosis. A review. Arch Fam Med 1:91-98

26. Huggett JF, McHugh TD, Zumla A (2003) Tuberculosis: amplification-based clinical diagnostic techniques. Int J Biochem Cell Biol 35:1407-1412

27. Mert A, Ozaras R, Tabak F et al (2003) Fever of unknown origin: a review of 20 patients with adult-onset Still's disease. Clin Rheumatol 22:89-93

28. Knockaert DC, Dujardin KS, Bobbaers HJ (1996) Long-term follow-up of patients with undiagnosed fever of unknown origin. Arch Intern Med 156:618-620 